\title{
Tetrandrine attenuates left ventricular dysfunction in rats with myocardial infarction
}

\author{
YOUYANG WU, WEI ZHAO, FANHAO YE, SHIWEI HUANG, HAO CHEN, RUI ZHOU and WENBING JIANG \\ Department of Cardiology, The Third Clinical Institute Affiliated to Wenzhou Medical University, \\ Wenzhou, Zhejiang 325000, P.R. China
}

Received March 16, 2020; Accepted September 16, 2020

DOI: $10.3892 / \mathrm{etm} .2020 .9551$

\begin{abstract}
The present study aimed to determine whether tetrandrine could attenuate left ventricular dysfunction and remodeling in rats with myocardial infarction. Sprague-Dawley rats were randomly divided into six groups ( $n=5 /$ group) as follows: i) Healthy control group; ii) sham operation group; iii) myocardial infarction model group; iv) myocardial infarction + low-dose tetrandrine group $(10 \mathrm{mg} / \mathrm{kg})$; v) myocardial infarction + medium-dose tetrandrine group $(50 \mathrm{mg} / \mathrm{kg})$; and vi) myocardial infarction + high-dose tetrandrine group $(80 \mathrm{mg} / \mathrm{kg})$. Left ventricular end-diastolic diameter (LVIDd), left ventricular end-systolic diameter (LVIDs), ejection fraction $(\mathrm{EF} \%)$ and left ventricular fractional shortening rate (FS\%) were measured using ultrasonography. The pathological changes were observed by hematoxylin and eosin (H\&E) staining. Left ventricular tissue section TUNEL staining was also performed. Furthermore, the triglyceride (TG), total cholesterol (TC), high density lipoprotein (HDL) and low-density lipoprotein (LDL) in the arterial blood were examined by biochemical testing. Expression levels of intracellular $\mathrm{Ca}^{2+}$ homeostasis-related proteins including ryanodine receptor calmodulin, CaM-dependent protein kinase II $\delta$, protein kinase A, FK506 binding protein 12.6 were measured using western blot analysis. Ultrasonography results showed that in the myocardial infarction model rats,
\end{abstract}

Correspondence to: Professor Wenbing Jiang, Department of Cardiology, The Third Clinical Institute Affiliated to Wenzhou Medical University, 57 Canghou Street, Wenzhou, Zhejiang 325000, P.R. China

E-mail: jiangwenbing163126@163.com

Abbreviations: LVIDd, left ventricular end-diastolic diameter; LVIDs, left ventricular end-systolic diameter; EF, ejection fraction; $\mathrm{H} \& \mathrm{E}$, hematoxylin and eosin; TG, triglyceride; TC, total cholesterol; HDL, high density lipoprotein; LDL, low density lipoprotein; RyR, ryanodine receptor; CaM, calmodulin; CaMKIIS, CaM-dependent protein kinase IID; PKA, protein kinase A; FKBP12.6, FK506 binding protein 12.6

Key words: myocardial infarction, tetrandrine, left ventricular remodeling, apoptosis, calcium homeostasis the levels of LVIDd and LVIDs were significantly higher; however, the levels of EF\% and FS\% were lower compared with those in the sham operation group, which was alleviated by tetrandrine. H\&E results showed that tetrandrine alleviated the pathological characteristics of myocardial infarction model rats. Furthermore, tetrandrine significantly inhibited myocardial cell apoptosis in rats with myocardial infarction. Tetrandrine significantly inhibited the levels of TG, TC and LDL and increased the levels of HDL in the arterial blood of rats with myocardial infarction. These findings revealed that tetrandrine could attenuate left ventricular dysfunction in rats with myocardial infarction, which might be associated with intracellular $\mathrm{Ca}^{2+}$ homeostasis.

\section{Introduction}

Coronary heart disease is a leading cause of death and long-term disability worldwide (1). Although important progress has been made in preventing coronary heart disease, the mortality rate still increased from 12.3 million in 1990 to 17.3 million in 2013 worldwide, with an increase of $41 \%$ (2). There is currently a lack of effective treatment available for patients with coronary heart disease and the 5-year mortality rate is as high as $50 \%$ (3). Myocardial infarction is defined as the death of cardiomyocytes caused by long-term ischemia (4). The main causes of death from myocardial infarction are progressive congestive heart failure, secondary severe arrhythmia, and sudden death (5). Therefore, there is a need to study new drugs for treating myocardial ischemia.

Tetrandrine, a bisbenzylisoquinoline alkaloid with a molecular formula of $\mathrm{C}_{33} \mathrm{H}_{42} \mathrm{~N}_{2} \mathrm{O}_{6}$, is the main biologically active ingredient extracted from the root of Stephania tetrandra S. Moore (6). It has been experimentally and clinically shown to have a variety of pharmacological effects, including muscle relaxation (7), allergy alleviation (8), antiarrhythmic (9), antihypertensive (10), antibacterial (11) and antitumor (12) properties and anticoagulation effects (13). In recent years, extensive and in-depth research into its pharmacological effects has been conducted. In terms of cardiovascular pharmacology, tetrandrine was found to be a natural non-selective calcium channel blocker and an antagonist of calmodulin (14). Previous studies have shown that tetrandrine has a better protective effect on myocardium compared with the sham operation group (15). It has been reported that tetrandrine can reduce the 
occurrence of ventricular arrhythmia in rats with myocardial ischemia-reperfusion, suggesting that it has a protective effect on arrhythmia caused by ischemia (16). However, to the best of our knowledge, so far, no study reported the role of tetrandrine in myocardial infarction. The current study hypothesized that tetrandrine may attenuate left ventricular dysfunction and remodeling in rats with myocardial infarction.

\section{Materials and methods}

Experimental groups. A total of 30 male Sprague-Dawley rats (weight, 200-250 g) from Shanghai Animal Research Center (http://www.slarc.org.cn/slarcWebSite/homeIndex. action) were randomly divided into six groups ( $\mathrm{n}=5 /$ group) as follows: i) Healthy control group; ii) Sham operation group; iii) Myocardial infarction model group; iv) myocardial infarction + low-dose tetrandrine group $(10 \mathrm{mg} / \mathrm{kg})$; v) myocardial infarction + medium-dose tetrandrine group $(50 \mathrm{mg} / \mathrm{kg})$; and vi) myocardial infarction + high-dose tetrandrine group $(80 \mathrm{mg} / \mathrm{kg})$, according to a previous study (17). All rats were housed at $21 \pm 2^{\circ} \mathrm{C}, 0.03 \% \mathrm{CO}_{2}, 30-70 \%$ relative humidity and $12 / 12 \mathrm{~h}$ light/dark cycle with free access to food and water. No rats died during the surgery. The present study was approved by the Ethics Committee of The Third Clinical Institute Affiliated to Wenzhou Medical University (Wenzhou, China).

Rat myocardial infarction model. Rats in the myocardial infarction model and sham operation groups were anesthetized with $3 \%$ sodium pentobarbital (Hangzhou Xiaoyong Biotechnology Co., Ltd.; $30 \mathrm{mg} / \mathrm{kg}$ ) by intraperitoneal injection. Subsequently, in order to fully expose the surgical area, the chest and axillary hair was shaved with a small animal shaver and disinfected with iodine and 75\% ethanol. Tracheal intubation was subsequently performed, and rats were operated on after confirming that they were unresponsive to pinching. After turning on the external light source and the microscope switch, the ventilator was turned on and the parameters (respiratory ratio, 2:1; tidal volume, $6-8 \mathrm{ml}$; frequency 70 times $/ \mathrm{min}$ ) were set. Subsequently, the rats were connected to a ventilator and observed for breathing. When the thoracic undulations and the ventilator frequency were consistent, the intubation was considered successful, indicating that myocardial infarction could be induced. Each rat was placed on the right side. The ophthalmic scissors were fixed under the axilla of the left forelimb and micro scissors were used to open the thorax between the third and fourth intercostal space to fully expose the heart. Rats in the sham operation group were sutured after thoracotomy. In order to fully expose the left anterior descending (LAD) coronary artery, micro straight forceps were used to gently pinch and tear pericardium under the left atrial appendage. Under the microscope, the direction or possible location of LAD was identified. A needle holder, 5-0 suture and a needle were used to apply a non-invasive suture next to the pulmonary artery cone below the root of the left atrial appendage to completely block LAD coronary artery blood flow for $3 \mathrm{~min}$. After the ligation was completed, 5-0 sutures were used to completely suture the thorax (seamless, no dislocation) and suture each layer of muscles and skin. After the surgery, the rats were observed for abnormal breathing. After the rats naturally awakened, the ventilator and tube were removed. Finally, the rats were reared normally as aforementioned. The tetrandrine groups were administered 10,50 or $80 \mathrm{mg} / \mathrm{kg}$ tetrandrine orally once a day at a fixed time starting from the second day after surgery by intragastric intubation. The rats in the sham operation and myocardial infarction groups were administered the same volume of normal saline in the same way. After 4 weeks, arterial blood was collected from each group. All rats were euthanized by $3 \%$ sodium pentobarbital $(150 \mathrm{mg} / \mathrm{kg})$ by intraperitoneal injection and the left ventricle tissue was removed. The present study complied with the Guide for the Care and Use of Laboratory Animals of the National Institutes of Health (18).

Rat myocardial infarction model and treatment effect evaluation. Rats in each group were evaluated for cardiac function by ultrasonography every 3 days for 4 weeks (18). From the ultrasound images, the left ventricular end-diastolic diameter (LVIDd) and left ventricular end-systolic diameter (LVIDs) were measured. In addition, the corresponding ejection fraction (EF\%) and left ventricular fractional shortening rate (FS\%) were automatically calculated with M-mode and 2D echocardiography (ACUSON Sequoia; Siemens Healthineers).

Hematoxylin and eosin $(H \& E)$ staining. Fresh myocardial tissue was fixed in $4 \%$ paraformaldehyde for $24 \mathrm{~h}$ at $37^{\circ} \mathrm{C}$. Subsequently, the tissue was dehydrated in ascending alcohol series. The fixed tissue was embedded in paraffin and cooled at $-20^{\circ} \mathrm{C}$. After the wax solidified, the wax block was removed from the embedding box and trimmed. The trimmed wax block was sliced to a thickness of $4 \mu \mathrm{m}$. Paraffin sections were subsequently stained. Briefly, paraffin sections were dewaxed (using xylene) into water. Sections were stained with Harris hematoxylin for $5-10 \mathrm{~min}$ at $37^{\circ} \mathrm{C}$. After rinsing with tap water, paraffin sections were differentiated with $1 \%$ hydrochloric acid alcohol for $10 \mathrm{sec}$. After $10 \mathrm{~min}$ of rinsing in tap water, paraffin sections were washed with PBS for $5 \mathrm{~min}$ at $37^{\circ} \mathrm{C}$. Then, the sections were stained with eosin staining solution for $1-3 \mathrm{~min}$ at $37^{\circ} \mathrm{C}$. After the slides were dehydrated, they were sealed with neutral gum and observed under an optical microscope (Olympus Corporation; magnification, $\mathrm{x} 50$ or $\mathrm{x} 400$ ).

TUNEL staining. Myocardial tissue sections were prepared as described in H\&E staining. TUNEL staining was performed using TUNEL apoptosis detection kit [cat. no. ATK00001; Pujian Biological (Wuhan) Technology Co., Ltd. (AtaGenix); http://www.atagenix.com/]. All experimental groups were incubated with $1 \mathrm{X}$ DNase I buffer for $10 \mathrm{~min}$. A TUNEL test solution was prepared according to the manufacturer's instructions. Each section was incubated with an appropriate amount of TUNEL detection solution for $60 \mathrm{~min}$ at $37^{\circ} \mathrm{C}$, and then $0.05 \mu \mathrm{g} / \mu 1$ of DAPI solution was incubated at room temperature for $10 \mathrm{~min}$ in the dark. Sections were immersed 3 times in PBS solution at room temperature for $5 \mathrm{~min}$ each time, and mounted with anti-fluorescence quenching mounting media [cat. no. ATK00001; Pujian Biological (Wuhan) Technology Co., Ltd. (AtaGenix)]. The results were observed under a fluorescence microscope (magnification, $\mathrm{x} 400$ ) in five random fields of view. The excitation wavelength range 
was $450-500 \mathrm{~nm}$, and the emission wavelength range was 515-565 nm.

Biochemical testing. The triglyceride (TG), total cholesterol (TC), high density lipoprotein (HDL) and low-density lipoprotein (LDL) levels in the arterial blood were examined using TG assay kit (cat. no. C061), TC determination kit (cat. no. C063), HDL assay kit (cat. no. K076) and LDL assay kit (cat. no. K075) (all from Changchun Huili Biotech Co., Ltd.; http://www.cchuili.com/).

Western blot analysis. The frozen myocardial tissue samples $\left(-20^{\circ} \mathrm{C}\right)$ were lysed on ice with RIPA lysis buffer (cat. no. P0013B; Beyotime Institute of Biotechnology) for $30 \mathrm{~min}$ at $4^{\circ} \mathrm{C}$ and centrifuged at $3,280 \mathrm{xg}$ at $4^{\circ} \mathrm{C}$ for $10 \mathrm{~min}$. The supernatant was then transferred to a new microcentrifuge tube. The protein concentration was measured by the BCA method (cat. no. P0009; Beyotime Institute of Biotechnology). A total of $20 \mu \mathrm{g}$ protein samples were used for SDS-PAGE and subsequently transferred to a PVDF membrane and blocked with 5\% non-fat milk-PBS solution at room temperature for $1 \mathrm{~h}$. The membrane was incubated with primary antibodies at $4^{\circ} \mathrm{C}$ overnight, followed by an incubation with horseradish peroxidase-conjugated secondary antibodies (1:5,000; cat. no. ab97040; Abcam) for $1 \mathrm{~h}$ at room temperature. Finally, protein bands were visualized by an ECL kit (Thermo Fisher Scientific, Inc.). The grayscale value was determined using ImageJ software (version 2.1.4.7; National Institutes of Health).

The primary antibodies $(1: 1,000)$ were as follows: Anti-ryanodine receptor (RyR2; cat. no. ab2868; Abcam); anti-phosphorylated (p)-RYR-2 (cat. no. ab59225; Abcam); anti-calmodulin (CaM; cat. no. sc-137079; Santa Cruz Biotechnology, Inc.); anti-CaM-dependent protein kinase IIס (CaMKIIo; cat. no. ab181052; Abcam); anti-protein kinase A (PKA; cat. no. sc-390548; Santa Cruz Biotechnology, Inc); anti-FK506binding protein 12.6(FKBP12.6; cat. no.bs-16093R; BIOSS); anti-cleaved-caspase3 (cat. no. ab49822; Abcam) and anti-pro-caspase3 (cat. no. ab32499; Abcam). $\beta$-actin (cat. no. bs-0061R; BIOSS) was used as an internal control.

Statistical analysis. All statistical analyses were performed using GraphPad Prism 7.0 (GraphPad Software, Inc.). Data are presented as the mean $\pm \mathrm{SD}$. Each experiment was repeated at least three times. Multiple comparisons were performed using one-way analysis of variance followed by Tukey's post hoc test. $\mathrm{P}<0.05$ was considered to indicate a statistically significant difference.

\section{Results}

Construction of a rat model of myocardial infarction. The LVIDd, LVIDs, EF\% and FS\% of rats in each group were measured using ultrasonography (Fig. 1A). The results showed that in the myocardial infarction model rats, the levels of LVIDd (Fig. 1B) and LVIDs (Fig. 1C) were significantly higher than those in the sham operation group. After treatment with different doses of tetrandrine, the levels of LVIDd and LVIDs were significantly decreased compared with those in the myocardial infarction model group. As shown in Fig. 1D and E, compared with the sham operation group, the levels of EF\% and FS\% were significantly lower in the myocardial infarction model group. However, $80 \mathrm{mg} / \mathrm{kg}$ tetrandrine treatment significantly increased the levels of EF\% and FS\% compared with the myocardial infarction model group. These results indicated that tetrandrine might alleviate myocardial infarction.

Tetrandrine alleviates the pathological characteristics of rat models of myocardial infarction. The H\&E results showed that the myocardial cells of rats in the healthy control group and sham operation group were neatly arranged, dense, complete and clear, with uniform intercellular spaces and low levels of extracellular matrix (Fig. 2). Compared with the sham operation group, increased volume of the surviving cardiomyocytes, loose and disordered cell arrangement, contracted or dissolved nuclei, widened intercellular space and broken or disappeared myocardial rhabdoms were observed in the myocardial infarction model group (Fig. 2). In the tetrandrine-treated group, the degree of myocardial infarction in rats was markedly reduced compared with the myocardial infarction model group in a dose-dependent manner, and the residual myocardium showed an island-like distribution (Fig. 2). These results revealed that tetrandrine could alleviate the pathological characteristics of myocardial infarction in rats.

Tetrandrine alleviates myocardial apoptosis in rats with myocardial infarction. The TUNEL results showed that almost no apoptotic cardiomyocytes in the healthy control group and sham operation group were observed, while there were numerous apoptotic cardiomyocytes in the myocardial infarction model group (Fig. 3). Tetrandrine (10, 50 and $80 \mathrm{mg} / \mathrm{kg}$ ) improved cardiomyocyte apoptosis in the myocardial infarction model. These results showed that tetrandrine alleviated myocardial apoptosis in rats with myocardial infarction.

Tetrandrine significantly inhibits the levels of $T G, T C$ and $L D L$ and increases the levels of $H D L$ in the arterial blood of rats with myocardial infarction. The TC, TG, LDL and HDL levels were measured in the arterial blood. The results showed that the levels of TC (Fig. 4A), TG (Fig. 4B) and LDL (Fig. 4C) in the arterial blood of rats with myocardial infarction were significantly higher than that in rats in the healthy control group and sham operation group. However, 50 and $80 \mathrm{mg} / \mathrm{kg}$ tetrandrine significantly inhibited the levels of TC, TG and LDL in the arterial blood of rats with myocardial infarction. Furthermore, the level of HDL in the arterial blood of rats with myocardial infarction was significantly lower than that in rats in the healthy control group and sham operation group. This effect was alleviated by 50 and $80 \mathrm{mg} / \mathrm{kg}$ tetrandrine (Fig. 4D).

Tetrandrine restores calcium homeostasis in rats with myocardial infarction. Western blotting was used to detect the levels of cleaved-caspase-3, pro-caspase-3, RyR2, p-RyR2, CaMKIII, PKA, CaM and FKBP12.6 in myocardial tissues of each group of rats (Fig. 5A). The results showed that there was no significant difference in cleaved-caspase-3, pro-caspase-3 and cleaved-caspase-3/pro-caspase-3 levels 
A
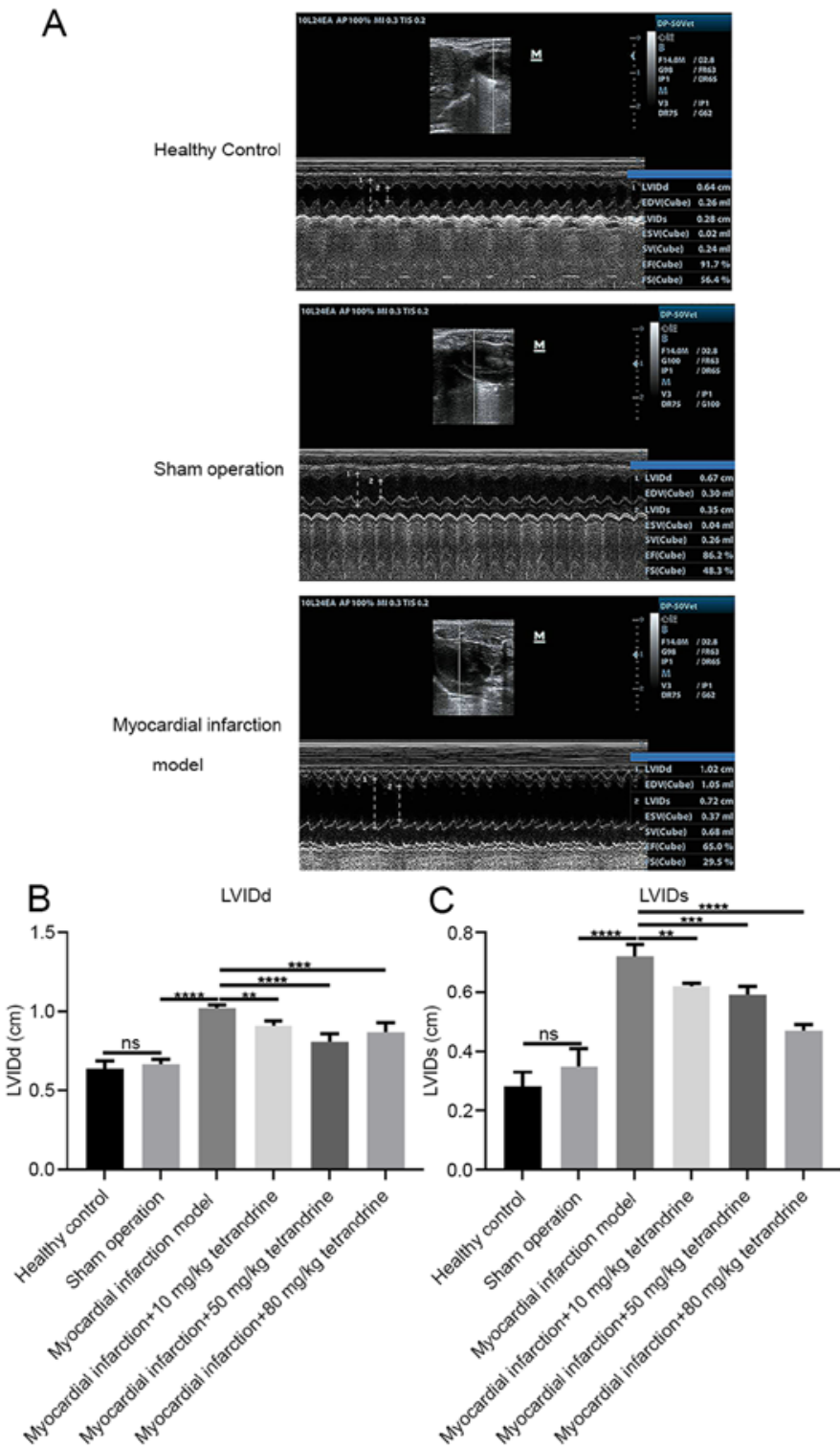
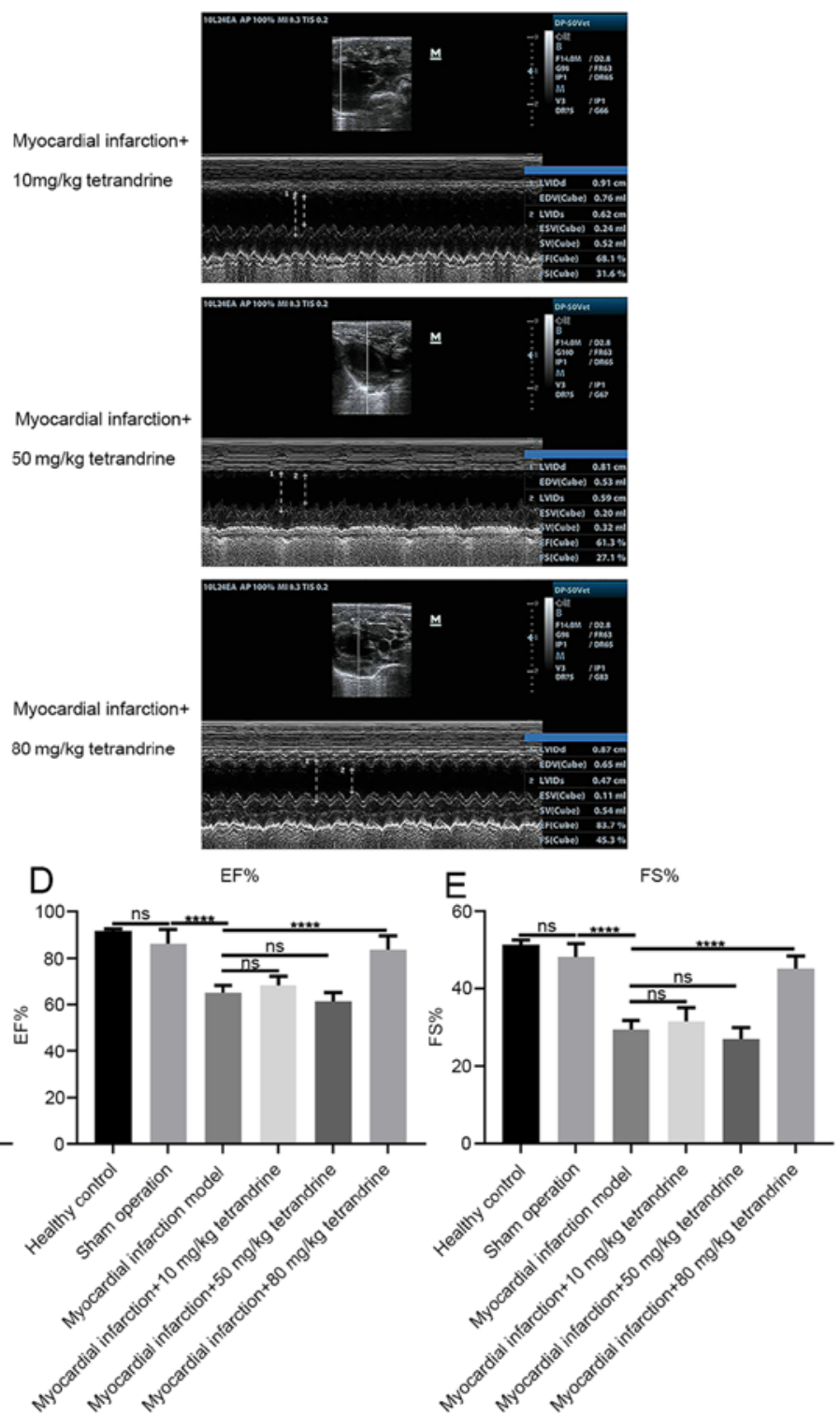

Figure 1. Construction of rat morels of myocardial infarction. (A) Representative images of ultrasonography. (B) LVIDd. (C) LVIDs. (D) EF\%. (E) FS\%. ${ }^{* *} \mathrm{P}<0.01 ;{ }^{* * *} \mathrm{P}<0.001$; and ${ }^{* * * *} \mathrm{P}<0.0001$. LVIDd, left ventricular end-diastolic diameter; LVIDs, left ventricular end-systolic diameter; EF\%, ejection fraction; FS\%, left ventricular fractional shortening rate; ns, no significant difference; M, M-mode ultrasonography.

between healthy control and sham operation groups (Fig. 5B-D). Furthermore, the level of RyR 2 and pro-caspase-3 was not different between the groups included in the present study. The expression level of cleaved-caspase-3 (Fig. 5B), the cleaved-caspase-3/pro-caspase-3 ratio (Fig. 5D) p-RyR2 (Fig. 5E), p-RyR2/RyR2 ratio (Fig. 5F), CaMKIId (Fig. 5G), PKA (Fig. 5H), and CaM (Fig. 5I) levels were significantly higher in the myocardial tissue of rats with myocardial infarction compared with the healthy control and sham operation groups. However, 50 and $80 \mathrm{mg} / \mathrm{kg}$ tetrandrine significantly inhibited the levels of cleaved-caspase-3, decreased the cleaved-caspase-3/pro-caspase-3 ratio, and inhibited the levels of p-RyR2, CaMKIIJ, PKA and CaM in the myocardial tissue of rats with myocardial infarction. As shown in Fig. 5J, in the myocardial tissues of rats with myocardial infarction, the expression level of FKBP12.6 was significantly lower than that in the healthy control and sham operation groups, and this effect was reversed by tetrandrine.

\section{Discussion}

A myocardial infarction model was successfully established in the current study. The findings revealed that tetrandrine could attenuate left ventricular dysfunction in rats with myocardial infarction by restoring calcium homeostasis. The present study provides a novel insight into the potential mechanisms of tetrandrine treatment of myocardial infarction.

To adapt to excessive heart pressure load, cardiac function is increased. The wall thickness and the stress of the left ventricle wall are increased to improve the contractile function of the heart, as a mechanism of early compensation (19). However, continuous pressure overload can promote myocardial hypertrophy, necrosis and apoptosis of myocardial cells, impair the contraction and/or diastolic function of the heart, and eventually develop into chronic heart failure or cause sudden cardiac death (19). In the present study, a 


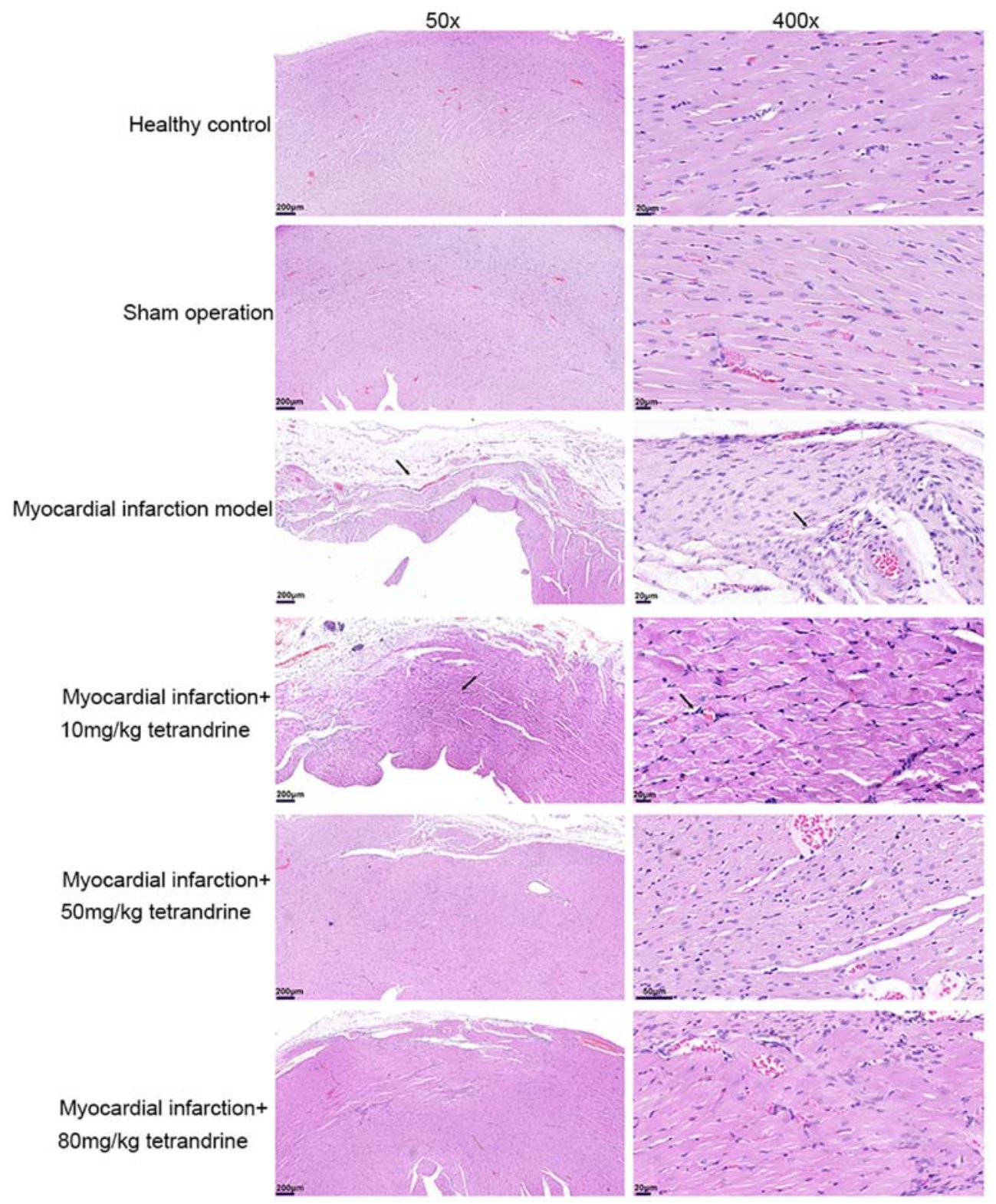

Figure 2. Tetrandrine alleviates the pathological characteristics of myocardial infarction in rats according to the hematoxylin and eosin staining results. Magnification, $\mathrm{x} 50$ or $\mathrm{x} 400$. There are five rats in each group. A representative image per group is shown. Arrows indicated the lesions.

myocardial infarction model was constructed by ligation of the left descending coronary artery in rats. Ultrasonography or hemodynamics can be used to detect and evaluate cardiac function (20). The current results showed that the levels of LVIDd and LVIDs were significantly higher and the levels of $\mathrm{EF} \%$ and $\mathrm{FS} \%$ were lower in the myocardial infarction model rats compared with the sham operation group, and these effects were alleviated by tetrandrine. Furthermore, the H\&E staining results showed that tetrandrine could alleviate the pathological characteristics of myocardial infarction model rats. TUNEL results showed that tetrandrine alleviated myocardial apoptosis in rats with myocardial infarction.

The potential mechanisms of tetrandrine treatment of myocardial infarction were further investigated. The protein levels of p-RyR2, CaMKIIS, PKA and CaM were significantly higher in the myocardial tissue of rats with myocardial infarction compared with the healthy control and sham operation groups, and these effects were reversed by tetrandrine. Furthermore, in the myocardial tissue of rats with myocardial infarction, the expression level of FKBP12.6 was significantly lower than that in the healthy control and sham operation groups, and this effect was reversed by tetrandrine. These results indicated that tetrandrine alleviated calcium homeostasis in rats with myocardial infarction. Previous studies have confirmed that the stability of the RyR is disrupted during heart failure due to sarcoplasmic reticulum diastolic $\mathrm{Ca}^{2+}$ leakage, which constitutes the myocardial cytological basis for diastolic dysfunction and fatal arrhythmia $(4,21)$. RyR can be divided into three subtypes and RyR2 is the only RyR type expressed in cardiomyocytes (22). $\mathrm{Ca}^{2+}$ leakage refers to the abnormal release of $\mathrm{Ca}^{2+}$ caused by an abnormal opening or incomplete closing of the RyR2 channel during diastole (23). The RyR2 channel is a large complex of signaling molecules composed of four isoform subunits with a molecular weight of $565 \mathrm{kDa}(24)$. There are also numerous accessory proteins that regulate 


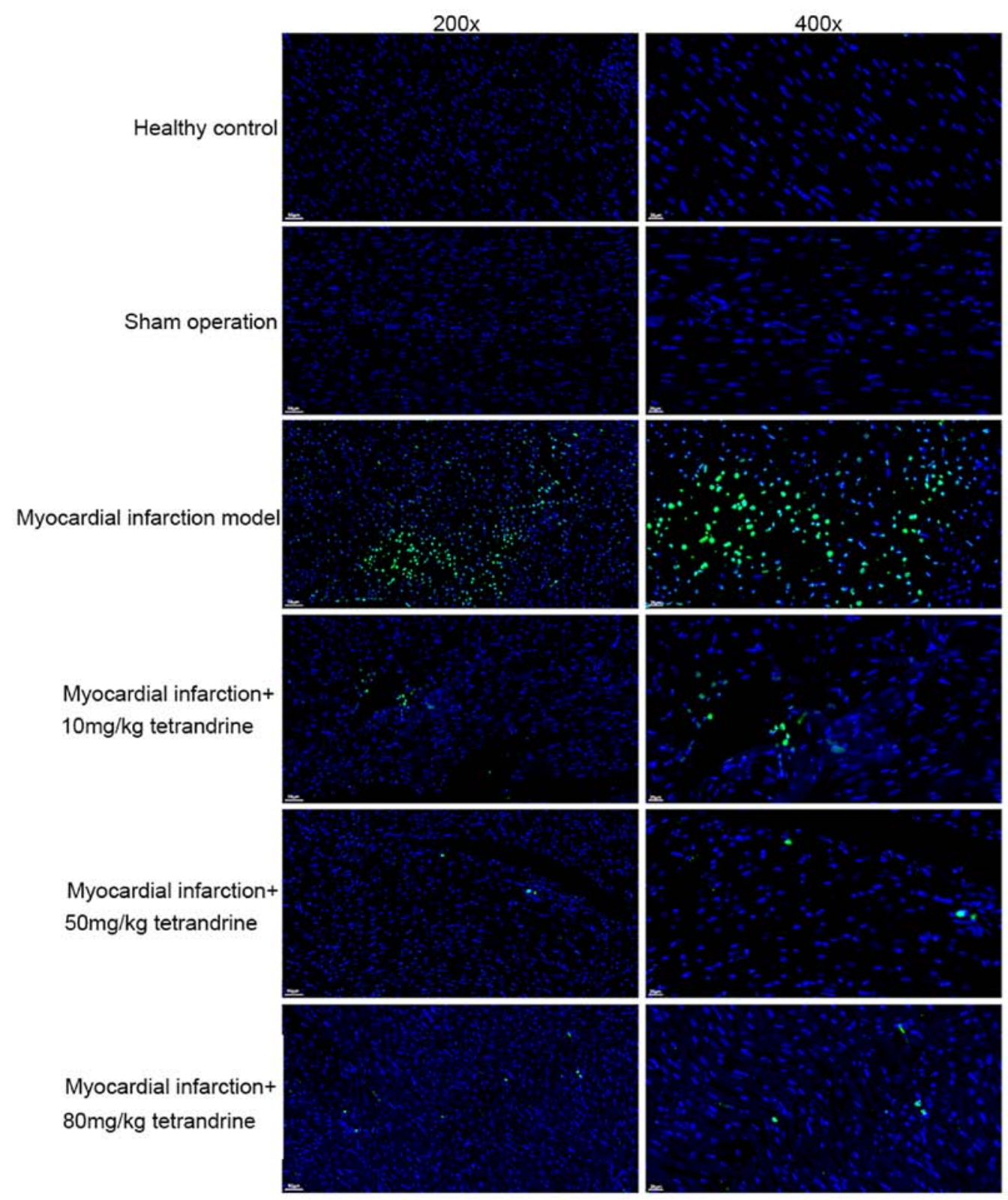

Figure 3. Tetrandrine alleviates myocardial apoptosis in rats with myocardial infarction, as demonstrated using TUNEL staining. Magnification, x50 or x400.

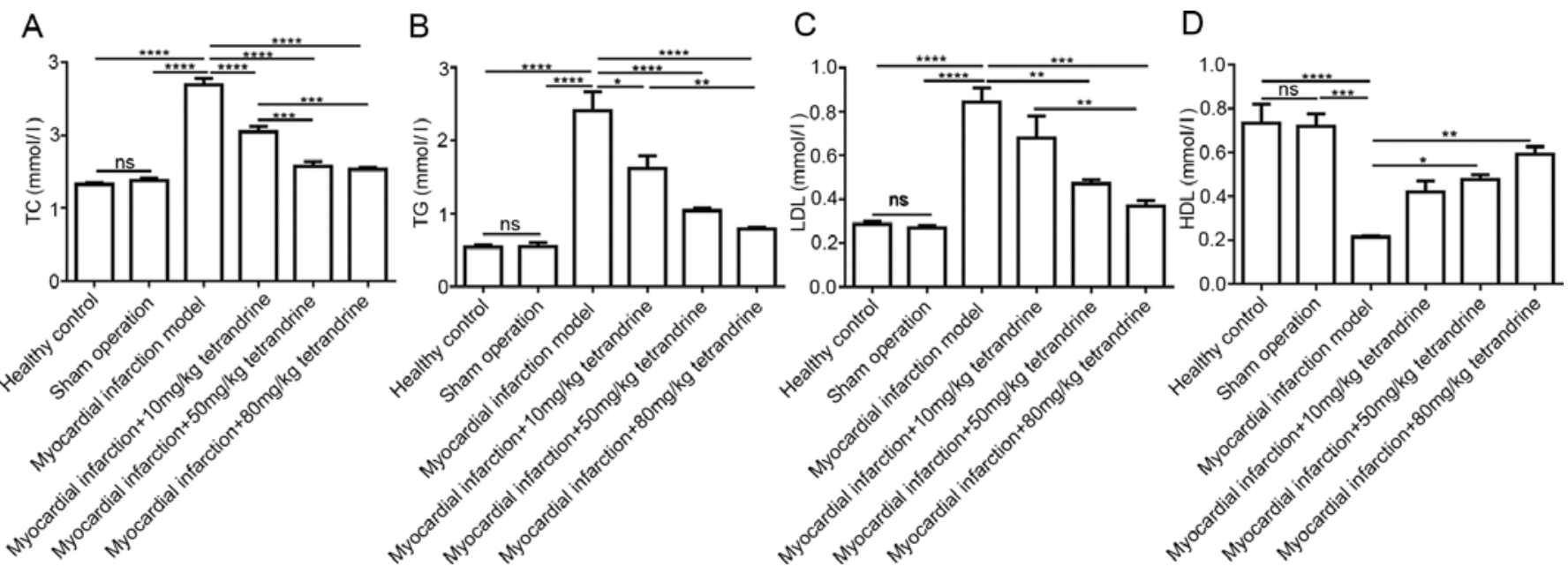

Figure 4. Tetrandrine significantly inhibits the levels of (A) TC, (B) TG and (C) LDL and increases the levels of (D) HDL in the arterial blood of rats with myocardial infarction. ${ }^{*} \mathrm{P}<0.05 ;{ }^{* * *} \mathrm{P}<0.01 ;{ }^{* * *} \mathrm{P}<0.001$; and ${ }^{* * * * *} \mathrm{P}<0.0001$. TG, triglyceride; TC, total cholesterol; HDL, high density lipoprotein; LDL, low density lipoprotein; ns, no significant difference. 


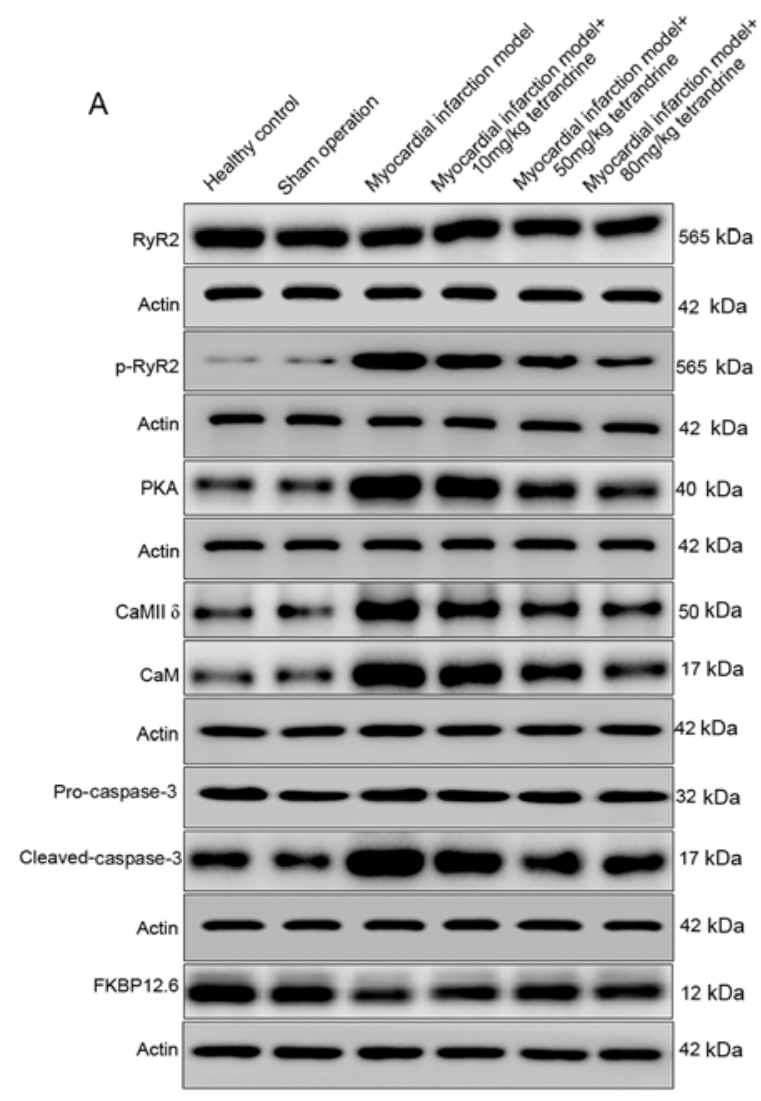

B
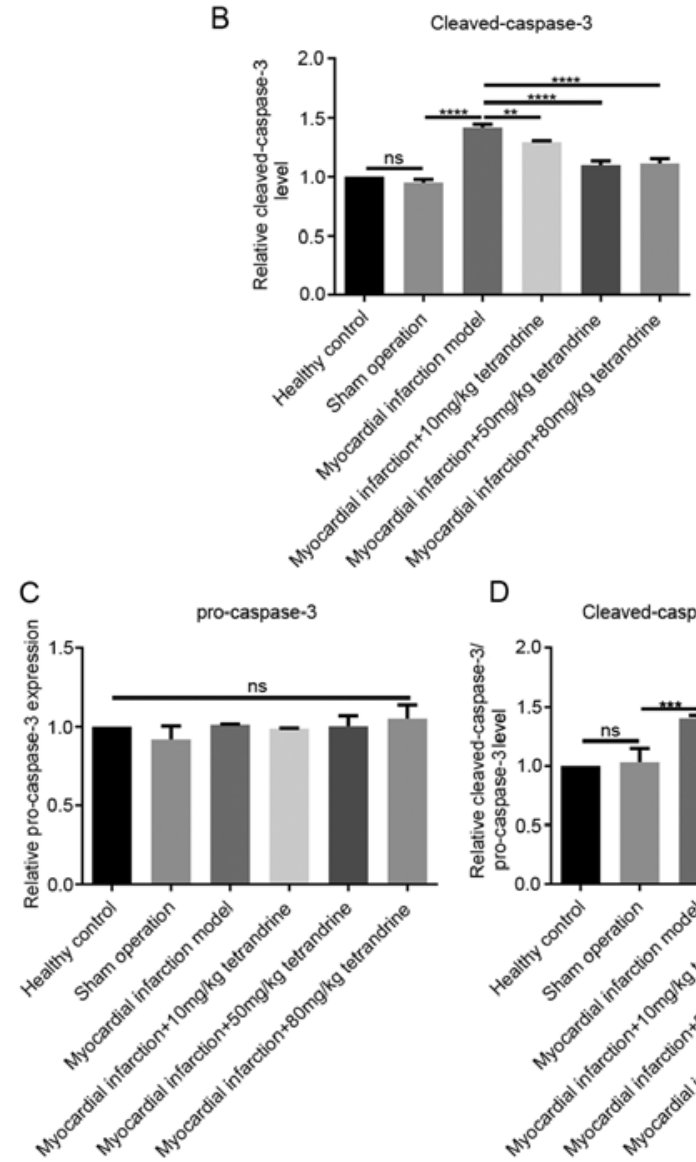

D Cleaved-caspase-3/pro-caspase-3

E
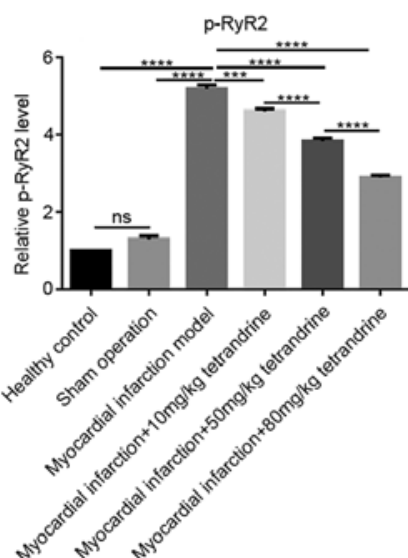

$\mathrm{H}$

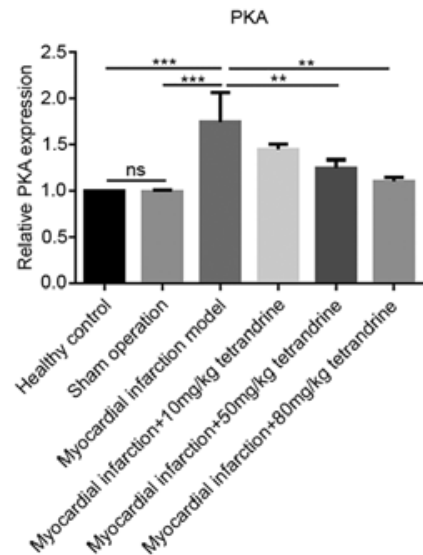

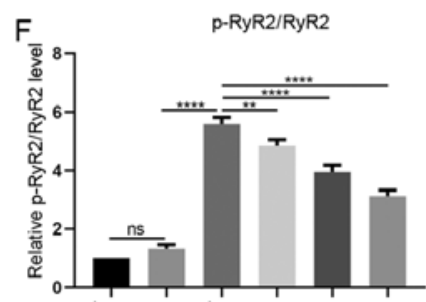

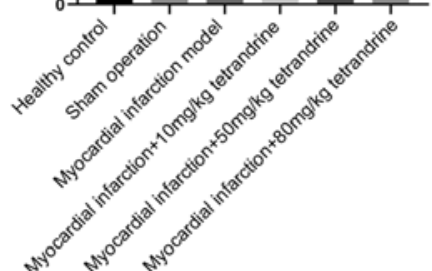

G

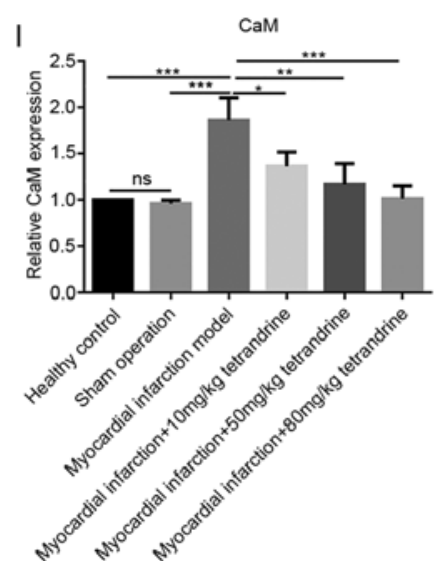

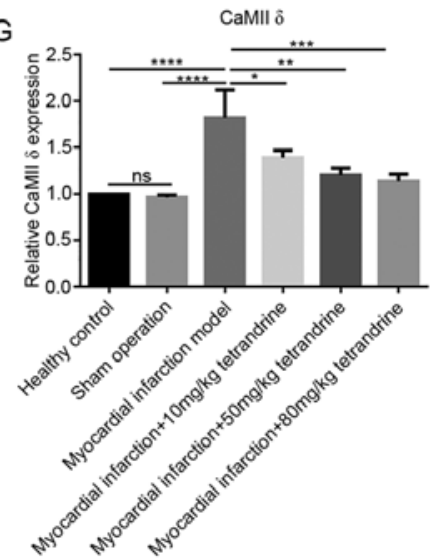
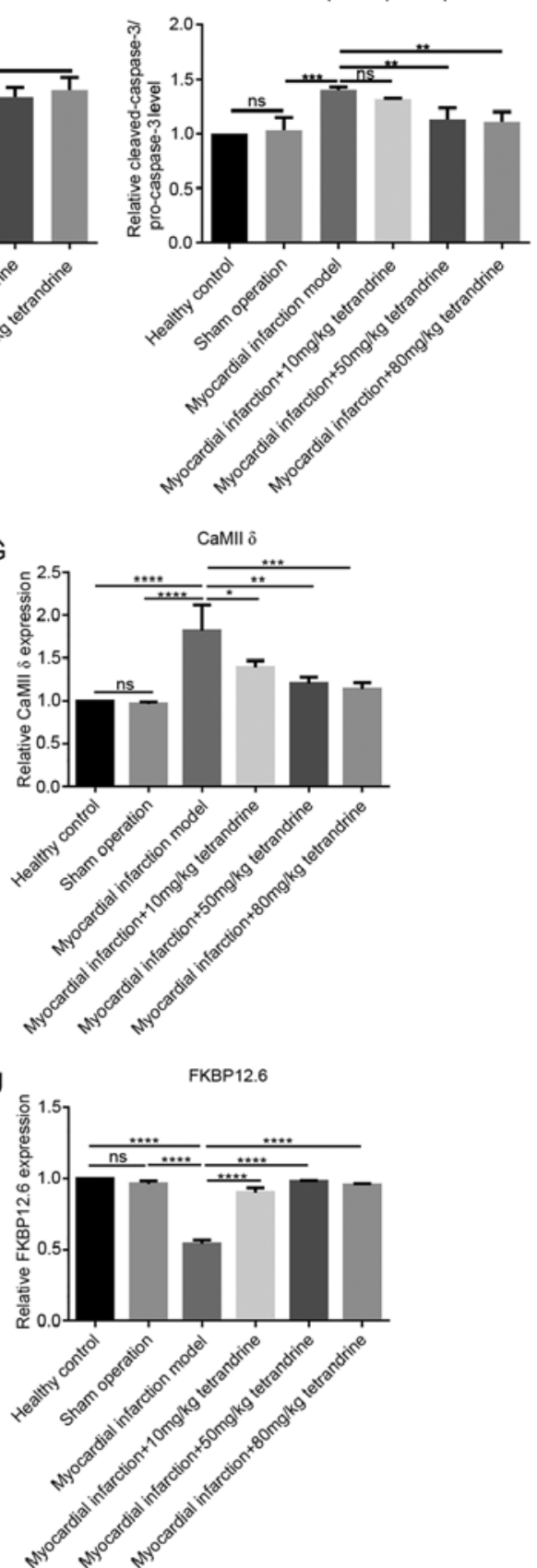

Figure 5. Tetrandrine restores calcium homeostasis in rats with myocardial infarction. (A) Representative images of western blotting. Protein levels of (B) cleaved-caspase-3, (C) pro-caspase3, (D) cleaved-caspase3/pro-caspase3 ratio, (E) p-RyR2, (F) p-RyR2/RyR2 ratio, (G) CaMKIID, (H) PKA, (I) CaM and (J) FKBP12.6 in myocardial tissue of each group of rats. ${ }^{*} \mathrm{P}<0.05 ;{ }^{* *} \mathrm{P}<0.01 ;{ }^{* * *} \mathrm{P}<0.001$; and ${ }^{* * * * *} \mathrm{P}<0.0001$. RyR, ryanodine receptor; CaM, calmodulin; CaMKIIס, CaM-dependent protein kinase IIס; PKA, protein kinase A; FKBP12.6, FK506 binding protein 12.6; p, phosphorylated; ns, no significant difference. 
the function of RyR2, including CaM, CaMKIIS, PKA and FKBP12.6. CaM is the main calcium-binding protein in cardiomyocytes (25). When $\mathrm{Ca}^{2+}$ increases in cardiomyocytes, $\mathrm{CaM}$ and $\mathrm{Ca}^{2+}$ combine to form a complex, which is activated and acts on the substrate protein CaMKIIס. PKA and CaMKIIS catalyze the phosphorylation of RyR2, and PP1 and PP2A catalyze the dephosphorylation of RyR2 (26). The combination of FKBP12.6 and RYR2 stabilizes the RYR2 channel complex. After RyR2 is phosphorylated, it separates from FKBP12.6 and opens. After dephosphorylation, it binds to FKBP12.6 and closes. In humans and rodents, Ser2808 and Ser2830 have been proposed to serve as the sites for PKA phosphorylation regulation of RyR2, and Ser2814 is a site for CaMKIIס phosphorylation regulation of RyR2 (27). Excessive phosphorylation of RyR2 or knockdown of FKBP12.6 in myocardial cells can separate RyR2 from FKBP12.6 and induce its opening, resulting in unstable RyR 2 channels and increased sensitivity to $\mathrm{Ca}^{2+}$ (28). Therefore, calcium homeostasis imbalance caused by $\mathrm{Ca}^{2+}$ leakage is an important cause of pathophysiological changes in myocardial failure (29). Considering the aforementioned mechanism of RyR 2 dysfunction in the pathogenesis of heart failure, RyR 2 is expected to become an important targeted therapy for heart failure (30). Promoting the interaction between FKBP12.6 and RyR2 and inhibiting PKA or CaMKII $\delta$-mediated RyR 2 hyperphosphorylation can enhance the stability of the RyR2 channel. Therefore, FKBP12.6, PKA and CaMKIId may become potential therapeutic targets for heart failure, which requires further research (31). According to previous reports, tetrandrine can inhibit extracellular $\mathrm{Ca}^{2+}$ influx, interfere with intracellular $\mathrm{Ca}^{2+}$ distribution, maintain intracellular $\mathrm{Ca}^{2+}$ homeostasis, and block various pathophysiological processes caused by abnormal calcium signaling (32). Tetrandrine can inhibit the increase of intracellular $\mathrm{Ca}^{2+}$ induced by $\mathrm{KCl}$ in a dose-dependent manner. In addition, its high concentration can also reduce the instantaneous increase in intracellular $\mathrm{Ca}^{2+}$ induced by caffeine (33). Tetrandrine can reduce diastolic blood pressure, ameliorate left ventricular hypertrophy and remodeling in hypertensive rats, reduce myocardial cell calcium overload, and increase myocardial myosin ATPase, $\mathrm{Na}^{+}-\mathrm{K}^{+}$-ATPase and $\mathrm{Ca}^{2+}$-ATP enzyme activity (34). Recent studies have reported that tetrandrine can reduce myocardial hypertrophy, ventricular enlargement and pulmonary congestion in a model of myocardial hypertrophy caused by aortic constriction $(35,36)$. It has been hypothesized that tetrandrine may act by inhibiting the activation of the oxygen free radical-dependent ERK1/2 signal transduction pathway (15). Previous studies have reported that the use of tetrandrine in rabbits after myocardial infarction induced by isoproterenol can reduce myocardial damage through antioxidative effects (35), and that tetrandrine has antioxidative and antifibrotic effects on the human heart, and improves ventricular compliance (36).

The present study further elucidated the mechanisms of ventricular myocyte calcium homeostasis and left ventricular remodeling in ischemic cardiomyopathy. By measuring the effect of tetrandrine on cardiac function in rats with ischemic cardiomyopathy, the results confirmed that tetrandrine could play a role in improving cardiac function in rats with ischemic cardiomyopathy. The effect of tetrandrine on the expression of regulatory proteins involved in ventricular calcium homeostasis in ischemic cardiomyopathy was also studied. However, further research is required to confirm this mechanism.

A myocardial ischemic injury model was established in the present study. The findings revealed that tetrandrine can protect the ischemic myocardium. In summary, previous research suggested that tetrandrine can have protective effects on ischemic heart, including reversing ventricular remodeling, reducing infarct size, inducing antioxidative and antifibrotic effects, improving cardiac function and reducing the occurrence of arrhythmia. The specific mechanism may be associated with the maintenance of intracellular $\mathrm{Ca}^{2+}$ homeostasis and antioxidative and antifibrotic effects.

\section{Acknowledgements}

Not applicable.

Funding

No funding was received.

\section{Availability of data and materials}

The datasets used and/or analyzed during the current study are available from the corresponding author on reasonable request.

\section{Authors' contributions}

WJ conceived and designed the study. YW, WZ and FY conducted most of the experiments, performed data analysis and wrote the manuscript. $\mathrm{SH}, \mathrm{HC}$ and $\mathrm{RZ}$ participated in the acquisition of data and helped draft the manuscript. All authors read and approved the final manuscript.

\section{Ethics approval and consent to participate}

The present study was approved by the Ethics Committee of The Third Clinical Institute Affiliated to Wenzhou Medical University (Wenzhou, China).

\section{Patient consent for publication}

Not applicable.

\section{Competing interests}

The authors declare that they have no competing interests.

\section{References}

1. Liao J, Wang J, Liu Y, Li J and Duan L: Transcriptome sequencing of lncRNA, miRNA, mRNA and interaction network constructing in coronary heart disease. BMC Med Genomics 12: $124,2019$.

2. Agha G, Mendelson MM, Ward-Caviness CK, Joehanes R, Huan TX, Gondalia R, Salfati E, Brody JA, Fiorito G, Bressler J, et al: Blood leukocyte DNA methylation predicts risk of future myocardial infarction and coronary heart disease. Circulation 140: 645-657, 2019. 
3. Zhou M, Bao Y, Li H, Pan Y, Shu L, Xia Z, Wu D, Lam KSL, Vanhoutte PM, Xu A, et al: Deficiency of adipocyte fatty-acid-binding protein alleviates myocardial ischaemia/reperfusion injury and diabetes-induced cardiac dysfunction. Clin Sci (Lond) 129: 547-559, 2015.

4. Thygesen K,AlpertJS and White HD; JointESC/ACCF/AHA/WHF Task Force for the Redefinition of Myocardial Infarction: Universal definition of myocardial infarction. J Am Coll Cardiol 50: 2173-2195, 2007.

5. Winkler A, Jaguś-Jamioła A, Uziębło-Życzkowska B, Orski Z, Krzyżanowski K, Smalc-Stasiak M and Kiliszek M: Predictors of appropriate interventions and mortality in patients with implantable cardioverter-defibrillators. Pol Arch Intern Med 129: 667-672, 2019.

6. Yuan X, Dou Y, Wu X, Wei Z and Dai Y: Tetrandrine, an agonist of aryl hydrocarbon receptor, reciprocally modulates the activities of STAT3 and STAT5 to suppress Th17 cell differentiation. J Cell Mol Med 21: 2172-2183, 2017.

7. Kwan CY, Ma FM and Hui SC: Inhibition of endothelium-dependent vascular relaxation by tetrandrine. Life Sci 64: 2391-2400, 1999.

8. Liou JT, Lin CS, Liao YC, Ho LJ, Yang SP and Lai JH: JNK/AP-1 activation contributes to tetrandrine resistance in T-cell acute lymphoblastic leukaemia. Acta Pharmacol Sin 38: 1171-1183, 2017.

9. Sakurai Y, Kolokoltsov AA, Chen CC, Tidwell MW, Bauta WE, Klugbauer N, Grimm C, Wahl-Schott C, Biel M and Davey RA: Ebola virus. Two-pore channels control Ebola virus host cell entry and are drug targets for disease treatment. Science 347 995-998, 2015.

10. Zhang J, Yu B, Zhang XQ, Sheng ZF, Li SJ, Wang ZJ, Cui XY, Cui SY and Zhang YH: Tetrandrine, an antihypertensive alkaloid, improves the sleep state of spontaneously hypertensive rats (SHRs). J Ethnopharmacol 151: 729-732, 2014.

11. Lee YS, Han SH, Lee SH, Kim YG, Park CB, Kang OH, Keum JH, Kim SB, Mun SH, Seo YS, et al: The mechanism of antibacterial activity of tetrandrine against Staphylococcus aureus. Foodborne Pathog Dis 9: 686-691, 2012.

12. Yu M, Liu T, Chen Y, Li Y and Li W: Combination therapy with protein kinase inhibitor $\mathrm{H} 89$ and Tetrandrine elicits enhanced synergistic antitumor efficacy. J Exp Clin Cancer Res 37: 114, 2018.

13. Westra IM, Oosterhuis D, Groothuis GM and Olinga P: Precision-cut liver slices as a model for the early onset of liver fibrosis to test antifibrotic drugs. Toxicol Appl Pharmacol 274 328-338, 2014

14. Ohta T, Yasuda W, Hasegawa A, Ito S and Nakazato Y: Effects of inhibitors for tyrosine kinase and non-selective cation channel on capacitative $\mathrm{Ca}(2+)$ entry in rat ileal smooth muscle. Eur J Pharmacol 387: 211-220, 2000.

15. Shen DF, Tang QZ, Yan L, Zhang Y, Zhu LH, Wang L, Liu C, Bian ZY and Li H: Tetrandrine blocks cardiac hypertrophy by disrupting reactive oxygen species-dependent ERK1/2 signalling. Br J Pharmacol 159: 970-981, 2010.

16. Ai J, Gao HH, He SZ, Wang L, Luo DL and Yang BF: Effects of matrine, artemisinin, tetrandrine on cytosolic $\left[\mathrm{Ca}^{2+}\right] \mathrm{i}$ in guinea pig ventricular myocytes. Acta Pharmacol Sin 22: 512-515, 2001

17. Pilz PM, Hamza O, Gidlöf O, Gonçalves IF, Tretter EV, Trojanek S, Abraham D, Heber S, Haller PM, Podesser BK and Kiss A: Remote ischemic perconditioning attenuates adverse cardiac remodeling and preserves left ventricular function in a rat model of reperfused myocardial infarction. Int J Cardiol 285 72-79, 2019.

18. Peng L, Zhao Y, Li Y, Zhou Y, Li L, Lei S, Yu S and Zhao Y: Effect of DJ-1 on the neuroprotection of astrocytes subjected to cerebral ischemia/reperfusion injury. J Mol Med (Berl) 97: 189-199, 2019.

19. Zhang TJ, Guo RX, Li X, Wang YW and Li YJ: Tetrandrine cardioprotection in ischemia-reperfusion (I/R) injury via JAK3/STAT3/Hexokinase II. Eur J Pharmacol 813: 153-160, 2017.
20. Sun M, Chen M, Dawood F, Zurawska U, Li JY, Parker T, Kassiri Z, Kirshenbaum LA, Arnold M, Khokha R and Liu PP: Tumor necrosis factor-alpha mediates cardiac remodeling and ventricular dysfunction after pressure overload state. Circulation 115: 1398-1407, 2007.

21. O'Brien F, Venturi E and Sitsapesan R: The ryanodine receptor provides high throughput $\mathrm{Ca}^{2+}$-release but is precisely regulated by networks of associated proteins: A focus on proteins relevant to phosphorylation. Biochem Soc Trans 43: 426-433, 2015.

22. Yano M, Kobayashi S, Kohno M, Doi M, Tokuhisa T, Okuda S, Suetsugu M, Hisaoka T, Obayashi M, Ohkusa T, et al: FKBP12.6-mediated stabilization of calcium-release channel (ryanodine receptor) as a novel therapeutic strategy against heart failure. Circulation 107: 477-484, 2003.

23. Landstrom AP, Dobrev D and Wehrens XHT: Calcium signaling and cardiac arrhythmias. Circ Res 120: 1969-1993, 2017.

24. Peng W, Shen H, Wu J, Guo W, Pan X, Wang R, Chen SR and Yan N: Structural basis for the gating mechanism of the type 2 ryanodine receptor RyR2. Science 354: aah5324, 2016.

25. Rocchetti M, Sala L, Dreizehnter L, Crotti L, Sinnecker D, Mura M, Pane LS, Altomare C, Torre E, Mostacciuolo G, et al: Elucidating arrhythmogenic mechanisms of long-QT syndrome CALM1-F142L mutation in patient-specific induced pluripotent stem cell-derived cardiomyocytes. Cardiovascular Res 113: 531-541, 2017

26. Fischer TH, Herting J, Tirilomis T, Renner A, Neef S, Toischer K, Ellenberger D, Förster A, Schmitto JD, Gummert J, et al: $\mathrm{Ca}^{2+} /$ calmodulin-dependent protein kinase II and protein kinase A differentially regulate sarcoplasmic reticulum $\mathrm{Ca}^{2+}$ leak in human cardiac pathology. Circulation 128: 970-981, 2013.

27. Huke S and Bers DM: Ryanodine receptor phosphorylation at Serine 2030, 2808 and 2814 in rat cardiomyocytes. Biochem Biophys Res Commun 376: 80-85, 2008.

28. MacMillan D: FK506 binding proteins: Cellular regulators of intracellular $\mathrm{Ca}^{2+}$ signalling. Eur J Pharmacol 700: 181-193, 2013.

29. Luo M and Anderson ME: Mechanisms of altered $\mathrm{Ca}(2)(+)$ handling in heart failure. Circ Res 113: 690-708, 2013.

30. Yamamoto $\mathrm{T}$, Yano $\mathrm{M}, \mathrm{Xu} \mathrm{X}$, Uchinoumi $\mathrm{H}$, Tateishi $\mathrm{H}$, Mochizuki M, Oda T, Kobayashi S, Ikemoto N and Matsuzaki M: Identification of target domains of the cardiac ryanodine receptor to correct channel disorder in failing hearts. Circulation 117: 762-772, 2008

31. Sag CM, Wagner S and Maier LS: Role of oxidants on calcium and sodium movement in healthy and diseased cardiac myocytes. Free Radic Biol Med 63: 338-349, 2013.

32. Huang T, Xu S, Deo R, Ma A, Li H, Ma K and Gan X: Targeting

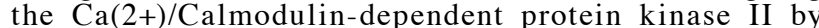
Tetrandrine in human liver cancer cells. Biochem Biophys Res Commun 508: 1227-1232, 2019.

33. Wang B and Xiao JG: Effect of tetrandrine on free intracellular calcium in cultured calf basilar artery smooth muscle cells. Acta Pharmacol Sin 23: 1121-1126, 2002.

34. Rao MR: Effects of tetrandrine on cardiac and vascular remodeling. Acta Pharmacol Sin 23: 1075-1085, 2002.

35. Pinelli A, Trivulzio S, Brenna S, Galmozzi G and Rossoni G: Pretreatment with tetrandrine has protective effects against isoproterenol-induced myocardial infarction in rabbits. In Vivo 24: 265-270, 2010.

36. Teng G, Svystonyuk D, Mewhort HE, Turnbull JD, Belke DD, Duff HJ and Fedak PWM: Tetrandrine reverses human cardiac myofibroblast activation and myocardial fibrosis. Am J Physiol Heart Circ Physiol 308: H1564-H1574, 2015.

This work is licensed under a Creative Commons Attribution-NonCommercial-NoDerivatives 4.0 International (CC BY-NC-ND 4.0) License. 